\title{
Clinically Significant Fatigue in Adult Leukemia Patients: Prevalence, Predictors, and Impact on Quality of Life
}

Isamme AlFayyad ${ }^{1}$, Mohamad Al-Tannir ${ }^{1}$, Muawiyah Yaqub ${ }^{2}$, Humariyah Heena ${ }^{3}$, Nawaf AlMukaibil ${ }^{4}$, Mohammed Ghazwani ${ }^{5}$, Amani Abu-Shaheen ${ }^{1}$

1. Epidemiology and Public Health, King Fahad Medical City, Riyadh, SAU 2. Oncology, King Fahad Medical City, Riyadh, SAU 3. Preventive Medicine, King Fahad Medical City, Riyadh, SAU 4. Internal Medicine, Al-Imam Mohammad Ibn Saud Islamic University, Riyadh, SAU 5. Internal Medicine, King Fahad Medical City, Riyadh, SAU

Corresponding author: Isamme AlFayyad, ialfayyad@kfmc.med.sa

\section{Abstract \\ Background}

Cancer-related fatigue (CRF) is a common distressing symptom in leukemia patients. CRF becomes clinically significant fatigue (CSF) when adversely affects health-related quality of life (HRQoL) and warrants further workup, referrals, and treatment. Objective: To assess the prevalence and predictors of CSF and assesses its impact on HRQoL in adult leukemia patients.

\section{Method}

Analysis was performed on 168 leukemia patients. The primary study outcomes were CSF (score $\geqslant 4$ ) as measured by the fatigue numerical rating scale and HRQoL using a validated Functional Assessment of Cancer Therapy-Leukemia (FACT-Leu) scale.

\section{Result}

The prevalence of CSF was 89 (53\%), with a mean score of $6.66 \pm 2.02$. About 106 (63.1\%) of leukemia patients had poor Health-related quality of life (HRQoL) (102.61 \pm 23.50$)$. Overall, FACT-Leu mean score indicated that study participants had poor HRQoL (114.70 \pm 29.67$)$. There was a statistically significant difference in HRQoL between the patients with CSF $104.89 \pm 28.82$ and Non-CSF $125.76 \pm 26.71$, $\mathrm{p}<0.001$. Poor appetite (odd ratio: 3.02 [95\% CI: 1.33-6.85]) was statistically significant predictors ( $\mathrm{p}<0.010)$ of CSF. Dependence on caregiver (odd ratio: 3.31 [95\% CI: 0.41-0.75]) and having non-CSF (odd ratio: 5.22 [95\% CI: 2.44-11.19]) were found statistically significant predictors of good HRQoL.

\section{Conclusion}

CSF is prevalent among leukemia patients, and adversely affects their HRQoL. Holistic assessment and supportive care are needed to reduce the burden of CSF and improve leukemia patients HRQoL.

Review began $12 / 06 / 2020$ Review ended 12/22/2020 Published 12/23/2020

\section{() Copyright 2020}

AlFayyad et al. This is an open access article distributed under the terms of the Creative Commons Attribution License CC-BY 4.0., which permits unrestricted use, distribution, and reproduction in any medium, provided the original author and source are credited.
Categories: Oncology, Hematology

Keywords: quality of life (qol), leukemia, clinically significant fatigue, cancer, adults

\section{Introduction}

Cancer-related fatigue (CRF) is the most prevalent, persistent, and distressing symptoms among cancer patients and survivors $[1,2]$. CRF is a debilitating symptom that interferes with physical and mental function and associated with reduced quality of life [3].

The prevalence of CRF is varied worldwide, reflecting the variety of the studied populations, the subjective nature of the cancer disease, and the treatment modalities, as well as the various used screening methods. CRF has been reported in patients with active chemotherapy between $59-90 \%$ and up to 100 in patients receiving radiotherapy and up to $25 \%$ among cancer survivors [4-7]. Among cancer diseases, CRF was experienced and frequently reported by leukemia patients than patients with solid tumors, with prevalence ranges between 33\% and 69\% [8,9]. Fatigue continues after the completion of the treatment course, roughly in a quarter of treated patients and increasing up to $35 \%$ of long-term cancer survivors [10]. The high prevalence of CRF indicates that symptom management is challenging and still not well managed in a remarkable proportion of cancer patients' life [3].

The clinical practice guidelines for CRF issued by the National Comprehensive Cancer Network (NCCN) represent the best "standard of care information" for the screening, assessment, and management of CRF [11]. These guidelines classified CRF into four clinically relevant subgroups according to their severity 
ratings to ensure that CRF is identified promptly and treated effectively. Understanding the relationship between the 4-subgroups and its clinically meaningful outcomes is significant for clinicians to inform and direct treatment decisions, promote clinician-patient dialogue on Clinically Significant Fatigue (CSF) management, and development of clinical practice guidelines [10]. The prevalence of CSF depends on the threshold score of severity (usually defined if the CRF a score of $\geqslant 4$ ) that is persistent, associated disability, warrants further workup, referrals, and treatment $[2,11]$.

The existing literature reported that risk factors associated with CRF are multifactorial. Some evidence suggests that CRF is associated with anemia, cachexia, neurological changes, infection, metabolic and endocrine disorders, psychological distress, concomitant medications, anti-neoplastic side-effects, pain, and paraneoplastic neurological syndromes [12]. Life prolongation, alleviation of the distressing symptoms, and preserving the optimal quality of life of cancer patients are the ultimate goals of cancer care [13]. However, CSF is associated with reduced cancer patients' health-related quality of life (HRQoL) at all stages of cancer and results in substantial adverse effects physical impairment, psychosocial distress, and economic burden for both patients and caregivers [14]. HRQoL data afford pertinent information for clinicians on the effectiveness of cancer treatment and are a crucial source of information for cancer patients to make informed decisions in their care plan [15].

As far as we know, there is a scarcity in the empirical evidence from developing countries on the prevalence of leukemia patients and its impact on HRQoL. Therefore, we aimed in this study to assess the prevalence and predictors of CSF and assess its impact of HRQoL in adult leukemia patients.

\section{Materials And Methods Study design and Setting}

A cross-sectional study was conducted in the comprehensive cancer centre (CCC) at King Fahad Medical City (KFMC), the largest tertiary care medical health care institution at the Saudi Ministry of Health.

\section{Study sample and sampling method}

A convenient sample of leukemia patients attending the outpatients' chemotherapy unit, hematology led clinic, or hospitalized as inpatient between May 2018 and January 2019 was approached by a trained study coordinator to participate in this study. We included patients aged 18 years and above, leukemia malignancy irrespective of their leukemia type, mentally competent, and free of preexisting psychiatric disorders. Patients with other hematological malignancies, unwilling to participate in the study were excluded.

\section{Sample size}

We used Cochran's method for estimating sample size with parameters of $95 \%$ confidence interval, $50 \%$ assumed prevalence of CRF and population size of 300 leukemia patients (cases on active treatment and follow-up in the CCC at KFMC in 2017); it was calculated that a minimum of 168 participants is required for this study.

\section{Survey tools}

The CRF was assessed using a numeric rating scale ( 0 - 10 points). We assessed the CRF severity as defined by the NCCN practice guidelines for cancer-related fatigue (0; no fatigue, 1-3; mild fatigue, 4-6; moderate fatigue, 7-10 severe fatigue), and CSF when the patient has fatigue $\geqslant 4$ [16]. HRQoL was evaluated using the Functional Assessment of Cancer Therapy-Leukemia (FACT-Leu), a 44-items measure of HRQoL and Leukemia specific symptoms utilizing essential customized questions of the Functional Assessment of Cancer Therapy-General (FACT-G), along with a cancer site-specific Leukemia subscale [17]. The FACT-G consists of 4 primary domains: Physical Well-being (PWB) (7-items; score range 0 to 28), Social/Family Wellbeing (SWB) (7-items; score range 0 to 28), Emotional Well-being (EWB) (6-items; score range 0 to 24), Functional Well-being (FWB) (7-items; score range 0 to 28). The additional Leukemia-Specific Subscale (LEUS) comprised of 17-items (score range o to 68) to evaluate specific concerns related to Leukemia [17].

We used the theoretical bio-psychosocial model adapted Hwang et al., to examine the effect of the sociodemographic characteristics variables, clinical relevant conditions variables, and biomedical parameters variables and their possible association as predictors for the study outcomes [18]. The examined sociodemographic characteristics included (age, gender, marital status, employment status, and need for caregiver), clinical relevant conditions (underlying chronic illness, time since diagnosis, relapsed disease, type of cancer treatment, sleeping hours, weight loss per kilogram within last 6-months, Eastern Cooperative Oncology Group Performance Status (ECOG-PS), dehydration, infection, and poor appetite), and biomedical parameters including Haemoglobin level ( $\mathrm{Hb}$ ), White Blood Cells (WBCs), sodium, Creatinine level, Calcemia, Potassium, Aspartate Transaminase (AST), total Bilirubin, Alanine Aminotransferase (ALT), Lactate Dehydrogenase (LDH). The cut-off values for the biomedical parameters (as approved by the laboratory lab at the study site) are described in table 1 . 


\section{Cureus}

\section{Data analysis}

Statistical analyses were done with IBM SPSS (Statistical Product and Service Solutions) Statistics (Version 24.0, Chicago). Descriptive analyses were used to summarize patients' characteristics (socio-demographic, clinical relevant conditions, biomedical parameters) and FACT-Leu Scale and subscale (means ants standard deviations). Univariate and multivariate regression models were developed to identify independent predictors of CSF and FACT-Lue. If the p-value was $<0.05$ in univariate models, the possible predictors were used in multiple logistic regression models. An independent sample t-test was used to compare the difference in the mean score of FACT-Leu scale and domains. Receiver Operating Characteristic (ROC) was run to determine the optimal cut-off score for good HRQoL.

\section{Ethical approval}

Approval for the study was granted from the institutional review board at KFMC. Written informed consent was obtained before patients participated in the study.

\section{Results}

One hundred and sixty-eight participants had completed the study survey. The study participants had an average of $43.99 \pm 19.20$ years of age. Most of the participants were males 103 (61.3\%), married 98 (58.7\%), and dependent on caregiver 110 (65.5\%). The mean time after diagnosis averaged $28.37 \pm 29.17$ months, and the mean for weight loss $(\mathrm{kg})$ averaged $-1.14 \pm 6.92$. The majority of the participants had chronic illnesses 107 (63.7\%) and on active treatment 127 (75.6\%). Poor appetite and sleeping < 7 hours was indicated by 101 (60.1\%) and 71 (42.3\%), respectively. The mean haemoglobin level and WBCs were 11.08 \pm 2.22 and 5.79 \pm 4.20 (Table 1).

Socio-demographic characteristics

Age (mean $\pm S D)$

$43.99 \pm 19.20$

Gender

Male

$103(61.3 \%)$

Female

65 (38.7\%)

Marital Status

Single

Married

$98(58.7 \%)$

Divorced

$8(4.8 \%)$

Widowed

Employed

Yes

No

$116(71.2 \%)$

Need a caregiver

Yes

No

58 (34.5\%)

Clinical relevant conditions

Underlying chronic illness

Yes

107 (63.7\%)

No

$61(36.3 \%)$

Time since Diagnosis

$28.37 \pm 29.17$

Relapsed disease

Yes

$39(23.4 \%)$

No

$128(76.6 \%)$

Active cancer treatment 


\section{Cureus}

Yes

No

Sleeping hours ( $<7$ hours)

Yes

No

Weight loss (mean per Kg)

ECOG-PS

0,1

$2,3,4$

Infection

Yes

No

Poor appetite

Yes

No

Biomedical parameters

$\mathrm{Hb}$ (Female $<11 \mathrm{~g} / \mathrm{dl}$, Male $<13.5 \mathrm{~g} / \mathrm{dl}$ )

WBCs $(>11 ; 10 \mathrm{e} 9 / \mathrm{L})$

Sodium (<135 mmol/L)

Creatinine (>90;umol/L)

Calcium (>2.5; $\mathrm{mmol} / \mathrm{L})$

Potassium (<3.4;mmol/L)

AST (>34;U/L)

Total Bilirubin (>20;umol/L)

ALT (>55;U/L)

LDH (>220;U/L)
$127(75.6 \%)$

$41(24.4 \%)$

$71(42.3 \%)$

$97(57.3 \%)$

$1.14 \pm 6.92$

106 (59.6\%)

$62(40.4 \%)$

21 (12.5\%)

147 (87.5\%)

$101(60.1 \%)$

67 (39.9\%)

$11.08 \pm 2.22$

$5.79 \pm 4.20$

$137.45 \pm 2.91$

$64.53 \pm 31.52$

$2.30 \pm 0.20$

$3.88 \pm 0.48$

$29.51 \pm 26.88$

$11.33 \pm 12.87$

$37.10 \pm 36.29$

$318.30 \pm 175.65$

TABLE 1: Bio-demographics of the study participants $(n=168)$.

ECOG-PS- Eastern Co-operative Oncology Group performance status; AST- aspartate transaminase, ALT- alanine aminotransferase, LDH- lactate dehydrogenase

CSF was present in 89 (53\%) patients with a mean of $6.66 \pm 2.02$, and 130 (77.4\%) of the patients reported CRF with a mean severity score of $5.12 \pm 2.86$. The four levels of CRF showed that the majority (34.6\%) of patients suffered from severe CRF, followed by moderate CRF 44 (33.9\%) (Table 2). 


\section{Cureus}

\begin{tabular}{|c|c|c|}
\hline & $n(\%)$ & Mean \pm SD \\
\hline \multicolumn{3}{|c|}{ Clinically significant fatigue } \\
\hline Yes & 89 (53) & $6.66 \pm 2.02$ \\
\hline No & $79(47)$ & $0.92 \pm 1.07$ \\
\hline Fatigue by (severity) & $130(77.4)$ & $5.12 \pm 2.86$ \\
\hline Mild & 41 (31.5) & $1.78 \pm 0.82$ \\
\hline Moderate & 44 (33.9) & $4.89 \pm 0.84$ \\
\hline Severe & $45(34.6)$ & $8.40 \pm 1.09$ \\
\hline
\end{tabular}

TABLE 2: Prevalence of CSF and CRF ( $n=168)$.

CSF- Clinically significant fatigue; CRF- Cancer related fatigue

Table 3 shows the univariate and multivariate associations of CSF in the study population. In the univariate analysis, increasing age, relapsed disease, losing weight, and poor appetite were significantly associated with a high prevalence of CSF $(\mathrm{p}<0.05)$. By multivariate analysis, poor appetite was the only significant factor associated with CSF. Patients with poor appetite were 3.02 folds more likely to exhibit CSF than patients who have a good appetite, and thus increasing the prevalence of CSF $(\mathrm{p}<0.001)$. 


\section{Cureus}

\begin{tabular}{|c|c|c|c|}
\hline & CSF group 89 (53) & Univariate analysis OR $[95 \% \mathrm{Cl}]$ & Multivariate analysis $\mathrm{OR}[95 \% \mathrm{Cl}]$ \\
\hline Age $($ mean $\pm S D)$ & $46.97 \pm 19.59$ & $0.98[0.97-0.99]^{*}$ & $0.99[0.97-1.00]$ \\
\hline Gender (Male) & $54(52.4 \%)$ & $1.06[0.57-1.97]$ & - \\
\hline \multicolumn{4}{|l|}{ Marital Status } \\
\hline Single & $23(46.9 \%)$ & 1 & \multirow{4}{*}{-} \\
\hline Married & $51(52 \%)$ & $3.39[0.82-14.06]$ & \\
\hline Divorced & $5(62.5 \%)$ & $2.76[0.71-10.83]$ & \\
\hline Widowed & $9(75 \%)$ & $1.80[0.26-12.50]$ & \\
\hline Employed & $22(46.8 \%)$ & $1.45[0.73-2.86]$ & - \\
\hline Needs a caregiver (Yes) & $62(56.4 \%)$ & $1.48[0.78-2.81]$ & - \\
\hline Underlying chronic illness (Yes) & $56(52.3 \%)$ & $0.93[0.49-1.75]$ & - \\
\hline Time since diagnosis & $29.47 \pm 28.30$ & $0.99[0.98-0.01]$ & - \\
\hline Relapsed disease & $26(66.7 \%)$ & $2.13[1.06-4.51]^{\star}$ & $1.83[0.82-4.06]$ \\
\hline Active cancer treatment & $70(55.1 \%)$ & $0.33[0.35-1.43]$ & - \\
\hline Sleeping hours (<7 hours) & $41(57.7 \%)$ & $0.92[0.39-1.33]$ & - \\
\hline Weight loss (mean per Kg) & $2.38 \pm 7.80$ & $1.07[1.01-1.13]^{\star}$ & 1 [0.95-1.07] \\
\hline ECOG-PS $(2,3,4)$ & $36(58.1 \%)$ & $1.38[0.74-2.61]$ & - \\
\hline Infection (Yes) & $12(57.1 \%)$ & $1.21[0.48-3.05]$ & - \\
\hline Poor appetite (Yes) & $64(63.4 \%)$ & $2.91[1.53-5.51]^{\star}$ & $3.02[1.33-6.85]^{\star}$ \\
\hline $\mathrm{Hb}$ (Female < $11 \mathrm{~g} / \mathrm{dll}$, Male < $13.5 \mathrm{~g} / \mathrm{dl})$ & $10.75 \pm 2.30$ & 1.16 [0.99-1.34] & - \\
\hline WBCs (>11;10e9/L) & $5.67 \pm 4.29$ & $1.02[0.94-1.09]$ & - \\
\hline Sodium (<135 mmol/L) & $137.48 \pm 3.02$ & $0.99[0.89-1.11]$ & - \\
\hline Creatinine (>90;umol/L) & $63.12 \pm 31.29$ & $1.00[0.99-1.01]$ & - \\
\hline Calcium (>2.5;mmol/L) & $2.28 \pm 0.17$ & $2.96[0.49-17.94]$ & - \\
\hline Potassium (<3.4;mmol/L) & $3.87 \pm 0.14$ & $1.14[0.59-2.19]$ & - \\
\hline AST (>34;U/L) & $31.55 \pm 27.11$ & $0.99[0.98-1.01]$ & - \\
\hline Total Bilirubin (>20;umol/L) & $11.57 \pm 13.19$ & $0.99[0.97-1.02]$ & - \\
\hline ALT (>55;U/L) & $38.49 \pm 32.37$ & 0.99 [0.99-1.01] & - \\
\hline $\mathrm{LDH}(>220 ; \mathrm{U} / \mathrm{L})$ & $309.05 \pm 141.72$ & $1.00[0.99-1.00]$ & - \\
\hline
\end{tabular}

TABLE 3: Univariate analysis for CSF ( $\geq 3 / 10$ ) and independent predictors of CSF by multivariate logistic regression analyses $(n=168)$.

Data presented either as number and percentage or mean and standard deviation

*Significant at $\mathrm{p}$-value $<0.005$.

ECOG-PS- Eastern Co-operative Oncology Group performance status; AST- aspartate transaminase, ALT- alanine aminotransferase, LDH- lactate dehydrogenase

Figure 1 displays the ROC curves for the FACT-Leu scale. The ROC curve (0.713) was statistically significant, $\mathrm{p}<0.003$. The cutoff point for FACT-Leu scale was found to be 126 out of 174 as the optimal cutoff point for good HRQoL. In the CSF group, 71 (79.8\%) had poor HRQoL, while 35 (44.3\%) of the non-CSF indicated poor HRQoL (Table 4). 


\section{Cureus}

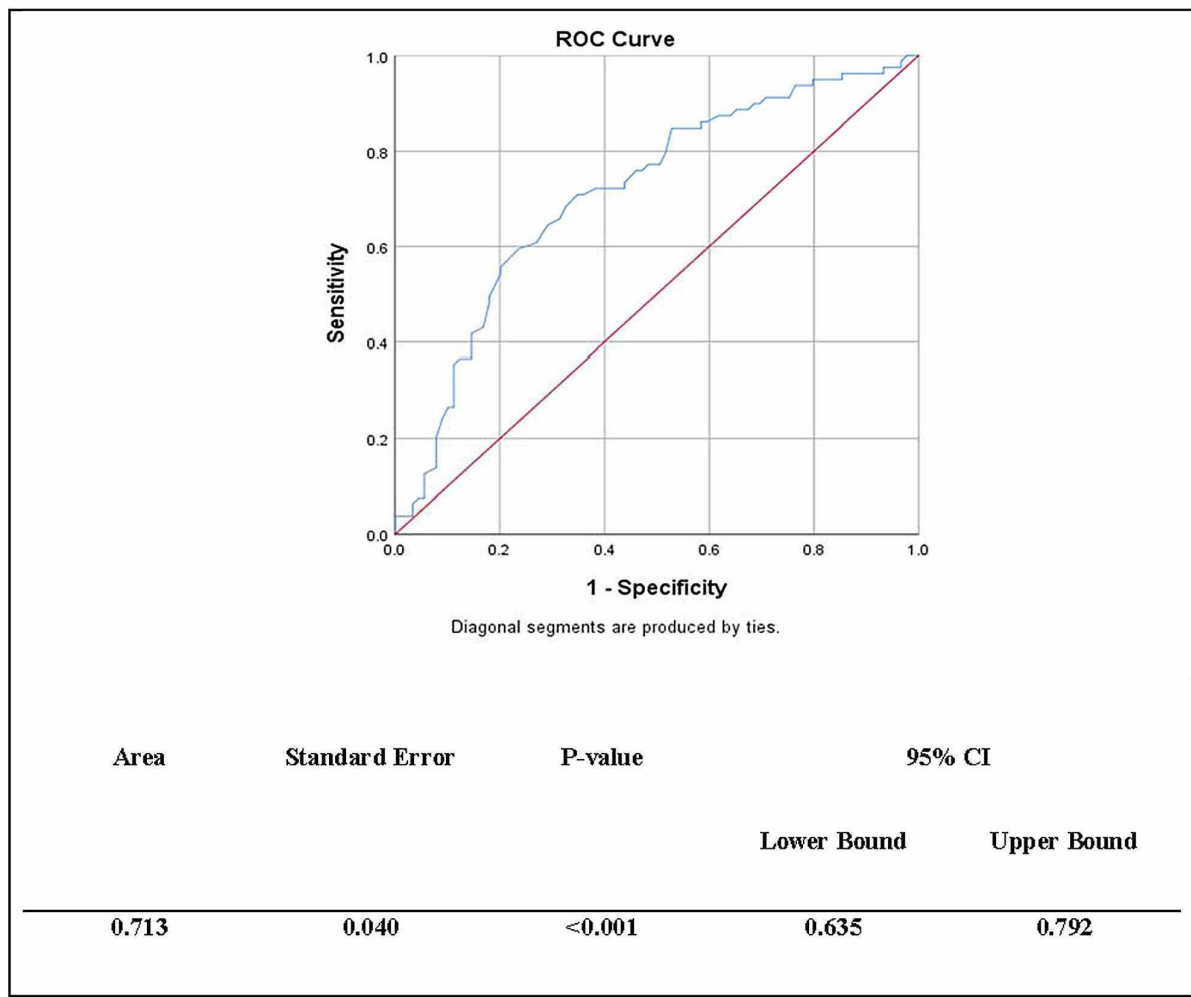

FIGURE 1: Receiver operating characteristic curve for FACT-Leu scale.

FACT-Leu- Functional Assessment of Cancer Therapy-Leukemia; ROC curve- receiver operating characteristic curve; $\mathrm{Cl}$ - Confidence interval

\begin{tabular}{|c|c|c|c|c|}
\hline & $n(\%)$ & Good HRQoL $(n=62)$ & Poor HRQoL $(n=106)$ & $\mathrm{p}$-value \\
\hline CSF group & $89(53)$ & $18(20.2 \%)$ & 71 (79.8\%) & \multirow{2}{*}{$<0.001$} \\
\hline Non-CSF group & 79 (47) & 44 (55.7\%) & 35 (44.3\%) & \\
\hline
\end{tabular}

TABLE 4: Distribution of good (FACT-Leu $\geq 126$ ) and poor (FACT-Leu <126) HRQoL among CSF and non-CSF patients.

*Significant at $\mathrm{p}$-value $<0.05$

FACT-Leu- Functional Assessment of Cancer Therapy-Leukemia; HRQoL- Health-related quality of life; CRF- Cancer-related fatigue; CSF- clinically significant fatigue

A statistically significant difference in the mean HRQoL was found between the CSF $104.89 \pm 28.82$ and nonCSF 125.76 \pm 26.71 groups ( $\mathrm{p}<0.001$ ); however, both groups had a poor HRQoL (FACT-Leu <126). Moreover, in the 4 HRQoL domains, there were statistically significant differences in the HRQoL between the patients with CSF and non-CSF, except in the social well-being ( $\mathrm{p}=0.239)$ (Table 5). 


\section{Cureus}

\begin{tabular}{|c|c|c|c|c|}
\hline & Total Mean \pm SD & non-CSF group Mean \pm SD & CSF group Mean \pm SD & P-value \\
\hline Total FACT-Leu scale & $114.70 \pm 29.67$ & $125.76 \pm 26.71$ & $104.89 \pm 28.82$ & $<0.001^{*}$ \\
\hline \multicolumn{5}{|l|}{ FACT-Leu domains } \\
\hline Physical well-being (0-28) & $16.40 \pm 7.76$ & $19.85 \pm 7.15$ & $13.38 \pm 7.02$ & $<0.001^{*}$ \\
\hline Social well-being (0-28) & $20.24 \pm 5.80$ & $19.68 \pm 6.72$ & $20.74 \pm 4.83$ & 0.239 \\
\hline Emotional well-being (0-24) & $17.26 \pm 5.18$ & $18.72 \pm 4.45$ & $15.97 \pm 5.45$ & $<0.001^{*}$ \\
\hline Functional well-being (0-28) & $17.70 \pm 6.90$ & $19.03 \pm 6.92$ & $16.55 \pm 6.70$ & $0.021^{*}$ \\
\hline LEUS $(0-68)$ & $43.40 \pm 13.88$ & $49.22 \pm 11.47$ & $38.25 \pm 13.85$ & $<0.001^{*}$ \\
\hline
\end{tabular}

TABLE 5: Mean FACT-Leu scale (HRQoL) and subscales.

*Significant at $\mathrm{p}$-value $<0.05$

FACT-Leu- Functional Assessment of Cancer Therapy-Leukemia; HRQoL- Health-related quality of life

The multivariate analysis showed that patients who were dependent form caregivers were 3.13 folds more likely to exhibit good HRQoL than patients who are independent on a caregiver. Moreover, patients who have non-CSF were 5.22 folds more likely to exhibit good HRQoL than patients who have CSF ( $\mathrm{p}<0.001)$ (Table 6$)$. 


\section{Cureus}

\begin{tabular}{|c|c|c|c|}
\hline & $\begin{array}{l}\text { Good HRQoL (FACT-Lue } \\
\geq 126)\end{array}$ & $\begin{array}{l}\text { Univariate analysis OR }[95 \% \\
\mathrm{Cl}]\end{array}$ & $\begin{array}{l}\text { Multivariate analysis OR }[95 \% \\
\mathrm{Cl}]\end{array}$ \\
\hline Age & $40.05 \pm 16.64$ & $0.98[0.97-0.99]^{*}$ & $0.99[0.97-1.01]$ \\
\hline Gender (Male) & 27 (26.2) & $1.24[0.65-2.37]$ & - \\
\hline \multicolumn{4}{|l|}{ Marital Status } \\
\hline Single & $20(40.8)$ & 1 & \multirow{4}{*}{-} \\
\hline Married & $38(38.8)$ & $3.45[0.68-17.45]$ & \\
\hline Divorced & $2(25)$ & $3.17[0.66-15.25]$ & \\
\hline Widowed & $2(16.7)$ & $1.17[1.84-15.13]$ & \\
\hline Non-employed & 25 (53.2) & $2.43[1.21-4.85]^{\star}$ & $0.74[0.32-1.72]$ \\
\hline Dependent form caregiver & $32(55.2)$ & $3.28[1.69-6.39]^{*}$ & $3.13[1.33-2.44]^{\star}$ \\
\hline Underlying chronic illness (No) & $25(41)$ & $1.31[0.69-2.51]$ & - \\
\hline Time since Diagnosis & $32.89 \pm 35.46$ & $1.01[0.99-1.02]$ & - \\
\hline Relapsed disease (No) & $53(41.4)$ & $2.36[1.03-5.37]^{*}$ & $1.32[0.51-3.44]$ \\
\hline Active cancer treatment (Yes) & 15 (36.6) & $0.98[0.47-2.04]$ & \\
\hline Sleeping hours (>7 hours) & $42(43.3)$ & $1.95[1.01-3.75]^{\star}$ & $2.14[0.98-4.69]$ \\
\hline Weight loss (mean per Kg) & $-0.60 \pm 6.24$ & $1.02[0.97-1.07]$ & - \\
\hline ECOG-PS $(0,1)$ & $44(41.5)$ & $1.74[0.89-3.39]$ & - \\
\hline Infection (No) & $59(40.1)$ & $4.02[1.13-14.27]^{*}$ & $3.56[0.89-14.32]$ \\
\hline Poor appetite (No) & $28(41.8)$ & $1.41[0.75-2.68]$ & - \\
\hline Non-CSF & $44(55.7)$ & $0.21[0.10-0.40]^{*}$ & $5.22[2.44-11.19]^{\star}$ \\
\hline $\begin{array}{l}\mathrm{Hb}(\text { Female }<11 \mathrm{~g} / \mathrm{dl} \text {, Male }<13.5 \\
\mathrm{g} / \mathrm{dl})\end{array}$ & $11.36 \pm 2.23$ & $1.10[0.95-1.27]$ & - \\
\hline WBCs (>11;10e9/L) & $5.03 \pm 3.58$ & $0.93[0.85-1.01]$ & - \\
\hline Sodium (<135 mmol/L) & $137.68 \pm 2.84$ & $1.04[0.93-1.17]$ & - \\
\hline Creatinine (>90;umol/L) & $65.60 \pm 26.11$ & $1.00[0.99-1.01]$ & - \\
\hline Calcium (>2.5;mmol/L) & $2.31 \pm 0.12$ & $2.25[0.41-12.33]$ & - \\
\hline Potassium (<3.4;mmol/L) & $3.92 \pm 0.38$ & $1.25[0.64-2.45]$ & - \\
\hline AST (>34;U/L) & $29.76 \pm 32.66$ & $1.00[0.99-1.01]$ & - \\
\hline Bilirubin Total (>20;umol/L) & $9.64 \pm 7.37$ & $0.98[0.95-1.01]$ & - \\
\hline ALT (>55;U/L) & $36.98 \pm 43.75$ & $1.00[0.99-1.01]$ & - \\
\hline Lactate Dehydrogenase (>220;U/L) & $296.28 \pm 198.42$ & $0.99[0.99-1.00]$ & - \\
\hline
\end{tabular}

\section{TABLE 6: Independent predictors of good HRQoL $(n=168)$.}

*Significant at $p$-value $<0.05$

ECOG-PS- Eastern Co-operative Oncology Group performance status; AST- aspartate transaminase, ALT- alanine aminotransferase, LDH- lactate dehydrogenase

\section{Discussion}

The current study findings highlight mainly two aspects of leukemia patients' care, fatigue, and HRQoL. Our results showed that leukemia patients had a high burden of CRF as reported by $77.4 \%$ of the study 
participants with a mean score of $5.12 \pm 2.86$. Overall, this prevalence estimate indicates that fatigue is prevalent among leukemia patients and is consistent with previously reported estimates among leukemia patients, which have varied between $27 \%$ and $92 \%[9,19,20]$.

The majority of our study participants had either severe or moderate CRF; hence, $68.4 \%$ of the patients were identified as having CRF of a severity level that was considered CSF with a mean score of $6.66 \pm 2.02$. A comparable result of CSF prevalence (62\%, weighted proportion) was reported by Alibhai et al. (n=13) [20]. However, our reported prevalence and mean of CSF were higher than the findings reported by Wang et al., (2002) findings which have shown that $53.5 \%$ (weighted proportion) leukemia patients $(n=106)$ had CSF with a mean score of $6.51 \pm 2.34$ [21]. Lacourt et al. has reported $41.8 \%$ (weighted proportion) of CSF among acute myeloid leukemia $(n=33)$ [22]. Furthermore, Romito et al. (2007) have reported a similar proportion $(63.4 \%)$ of general cancer patients experienced CSF [8].

Our study findings indicated that ageing was not a predictor of CSF, which was consistent with previous studies in haematology malignancy patients [20,21]. In this study, other socio-demographic characteristics, including gender, marital status, and employment, need for a caregiver, and monthly income were also not predictive variables of CSF, which were consistent with Wang's (2002) study [21].

In this study, evidence of chronic illness, weight loss, disease duration, active cancer treatment, ECOG-PS status, infection, and difficulty in sleeping ( $<7$ hours) was not associated with CSF. A previous study indicated that the high prevalence and exacerbation of fatigue severity in cancer patients are demonstrated by disease progression [21]. However, ECOG-PS was not retained as a predictor of fatigue in our study.

Sleep and disturbance CSF have been documented as concurrent symptoms in leukemia patients [22]. Inconsistence with our results, previous literature demonstrated that insufficient sleep was significantly associated with CSF [21]. Furthermore, our results were consistent with earlier reports that poor appetite predicts and intensify CSF in patients with leukemia [23].

The association of anemia with CSF is controversial. Anemia could be attributed to the cancer disease itself, the myelosuppressive treatment, blood loss, or bone marrow infiltration [24]. In this study, anemia was not a predictor of CSF. Similarly, some studies have demonstrated that anemia is not associated with CSF [20,21]. The reason that anemia was not a predictor of fatigue in this study is most probably that anemia was transient and treated supportively, which might limit the effect of this variable on CSF among the study participants.

Fatigue was reported as a significant predictor of impaired HRQoL and poor survival in leukemia patients [20]. Fatigue can adversely disturb patients' adherence to the treatment regime and can be doselimiting [25]

The ROC analysis showed that the cut-off score for good HRQoL in our study sample (FACT-Lue) was $\geqslant 126$ of 174. Overall, HRQoL mean score (104.89 \pm 28.82$)$ assessed by FACT-Leu was below the optimal cut-off score indicating poor HRQoL among our leukemia patients. The results of HRQoL employing FACT-Leu reported in the literature were varied. These variances could be attributed to measuring a specific leukemia disease instead of all leukemia diseases, sample size, the phase of the disease, and type of treatment and study settings. In our discussion, we will compare our findings with studies using the FACT-Leu scale to eliminate measurement bias. Mamolo et al. found that adults with newly diagnosed or relapsed/refractory de novo Acute Myeloid Leukemia (AML) $(n=68)$ had a poor HRQoL (FACT-Leu score: 100 \pm 24.0$)$ [26]. Albrecht et al. had reported the HRQoL experienced by newly diagnosed AML patients during chemotherapy induction was at baseline and up to six weeks during the chemotherapy (FACT-Leu score: $112.39 \pm 22.11$ ) [27]. Tinsley et al. had reported as well a poor HRQoL among AML patients during the intensive treatment (FACT-Leu score: 118.4 \pm 24.9 ) and non-intensive treatment (FACT-Leu score: $120.3 \pm 24.8$ ) [28]. Moreover, Kayastha et al. indicated that relapsed AML patients had poor HRQoL (FACT-Leu score=113.4 \pm 37.0 ) [29]. Remarkably, the FACT-Leu mean score of the CSF $(104.89 \pm 28.82)$ and non-CSF $(125.76 \pm 26.71)$ patients indicated a poor HRQoL (<126 of 174) among our study participants.

Striving with physical activity and psychosocial aspects, living with ambiguity, fear about future and disease relapse and effect on daily life are evident in hematologic malignancies patients, including leukemia. In the present study, FACT-Leu subscales mean scores differentiated showed that the patients with CSF had worst HRQoL than patients with non-CSF, except with SWB. Similar results were observed in the study performed by Kayastha et al., in the PWB score [29]. However, our study patients showed better values in the SWB, EWB, FWB, and LEUS scores in comparison to Kayastha et al., and Mamolo et al., [26,29]. The higher level of SWB in the current study may perhaps reflect the levels of social support given to leukemia patients at the study settings. Overall, these results figure out the need to enhance the whole status of patients with Leukemia, in particular, those who indicated physical impairment.

Several variables have been identified in the multivariate regression model. A caregiver plays a crucial role in patients' management of their illness. They are often the primary source of physical, social, and emotional support for the patient. Our results support this by asserting that patients who are independent of 
caregivers' support are less likely to have better HRQoL than patients who have a caregiver. Distressing symptoms experienced by leukemia patients like disturbed sleeping disrupt their HRQoL. Therefore, sufficient sleeping hours predict better HRQoL. Fatigue is associated with declines in all subscales of FACTLeu scale and adversely interferes with the physical and psychosocial domains of HRQoL [30]. Because fatigue among cancer patients, is inevitable, extensive efforts and strategies shall be made to reduce fatigue level to the mild levels. Identification of fatigue and HRQoL could lead to the development of supportive care programs based on a patient-centred approach and experience.

Several limitations should be considered when interpreting our data. The study sample size was modest. The cross-sectional design does not provide definite causation between fatigue and HRQOL among leukemia patients. Our study was conducted in a single institution; although the largest in Saudi Arabia, our data generalizability might be threatened. Our study did not identify fatigue severity and patients' quality of lifebased on the type of leukemia or type of treatment.

\section{Conclusions}

CSF is widespread among patients with leukemia. It is essential to identify the prevalence and the predictors of CSF and HRQoL to develop a patient-centred supportive care program. Our study findings support the importance of adapting NCCN guidelines to screen, evaluate, and manage CSF. Oncologists should be aware of CSF and address it throughout the continuum of leukemia treatment.

\section{Additional Information \\ Disclosures}

Human subjects: Consent was obtained by all participants in this study. King Fahad Medical City issued approval 17-442. Animal subjects: All authors have confirmed that this study did not involve animal subjects or tissue. Conflicts of interest: In compliance with the ICMJE uniform disclosure form, all authors declare the following: Payment/services info: All authors have declared that no financial support was received from any organization for the submitted work. Financial relationships: All authors have declared that they have no financial relationships at present or within the previous three years with any organizations that might have an interest in the submitted work. Other relationships: All authors have declared that there are no other relationships or activities that could appear to have influenced the submitted work.

\section{References}

1. Mustian KM, Alfano CM, Heckler C, et al.: Comparison of pharmaceutical, psychological, and exercise treatments for cancer-related fatigue: a meta-analysis. JAMA Oncol. 2017, 1:961-8. 10.1001/jamaoncol.2016.6914

2. Berger AM, Mitchell SA, Jacobsen PB, Pirl WF: Screening, evaluation, and management of cancer-related fatigue: ready for implementation to practice?. CA Cancer J Clin. 2015, 65:190-211. 10.3322/caac.21268

3. Teunissen SC, Wesker W, Kruitwagen C, De Haes HC, Voest EE, De Graeff A: Symptom prevalence in patients with incurable cancer: a systematic review. J Pain Symptom Manage. 2007, 34:94-104. 10.1016/j.jpainsymman.2006.10.015

4. Chang YD, Smith J, Portman D, Kim R, Oberoi-Jassal R, Rajasekhara S, Davis M: Single institute experience with methylphenidate and American ginseng in cancer-related fatigue. Am J Hosp Palliat Care. 2018, 35:144-50. 10.1177/1049909117695733

5. Donovan KA, McGinty HL, Jacobsen PB: A systematic review of research using the diagnostic criteria for cancer-related fatigue. Psychooncology. 2013, 22:737-44. 10.1002/pon.3085

6. Alexander S, Minton O, Stone PC: Evaluation of screening instruments for cancer-related fatigue syndrome in breast cancer survivors. J Clin Oncol. 2009, 27:1197-201. 10.1200/JCO.2008.19.1668

7. Servaes P, Verhagen C, Bleijenberg G: Fatigue in cancer patients during and after treatment: prevalence, correlates and interventions. Eur J Cancer. 2002, 38:27-43. 10.1016/s0959-8049(01)00332-X

8. Romito F, Montanaro R, Corvasce C, Di Bisceglie M, Mattioli V: Is cancer-related fatigue more strongly correlated to haematological or to psychological factors in cancer patients?. Support Care Cancer. 2008, 16:943-6. 10.1007/s00520-007-0357-1

9. Zordan R, Manitta V, Nandurkar H, Cole-Sinclair M, Philip J: Prevalence and predictors of fatigue in haemooncological patients. J. Intern. Med.. 2014, 44:1013-7. 10.1111/imj.12517

10. Wang XS, Zhao F, Fisch MJ, O'Mara AM, Cella D, Mendoza TR, Cleeland CS: Prevalence and characteristics of moderate to severe fatigue: a multicenter study in cancer patients and survivors. Cancer. 2014 , 120:42532. 10.1002/cncr.28434

11. Mock V, Atkinson A, Barsevick A, et al.: NCCN Practice guidelines for cancer-related fatigue. Oncology. 2000, 14:151-61.

12. Bower JE: Cancer-related fatigue-mechanisms, risk factors, and treatments . Nat Rev Clin Oncol. 2014, 11:597. 10.1038/nrclinonc.2014.127

13. Korfage I, Rietjens, J. Van der Heide A: ADVANCE-advance care planning; an innovative palliative care intervention to improve quality of life in cancer patients-a multi-centre cluster randomized clinical trialfp7 project. Impact. 2017, 4:37-39. 10.21820/23987073.2017.4.37

14. Yeo TP, Cannaday S: Cancer-related fatigue: impact on patient quality of life and management approaches . Nursing: research and reviews. 2015, 5:65. 10.2147/NRR.S41957

15. Bottomley A, Aaronson NK: International perspective on health-related quality-of-life research in cancer 
clinical trials: the European organisation for research and treatment of cancer experience. J Clin Oncol. 2007, 25:5082-6. 10.1200/JCO.2007.11.3183

16. Gerber LH, Stout N, McGarvey C, et al.: Factors predicting clinically significant fatigue in women following treatment for primary breast cancer. Support Care Cancer. 2011, 19:1581-91. 10.1007/s00520-010-0986-7

17. Cella D, Jensen SE, Webster K, et al.: Measuring health-related quality of life in leukemia: the functional assessment of cancer therapy-leukemia (FACT-Leu) questionnaire. Value Health. 2012, 15:1051-1058. 10.1016/j.jval.2012.08.2210

18. Hwang SS, Chang VT, Rue M, Kasimis B: Multidimensional independent predictors of cancer-related fatigue . J Pain Symptom Manage. 2003, 26:604-14. 10.1016/s0885-3924(03)00218-5

19. Musarezaie A, Khaledi F, Esfahani HN, Ghaleghasemi TM: Factors affecting quality of life and fatigue in patients with leukemia under chemotherapy. J Educ Health Promot. 2014, 3:64. 10.4103/2277-9531.134778

20. Alibhai SM, Leach M, Kowgier ME, Tomlinson GA, Brandwein JM, Minden MD: Fatigue in older adults with acute myeloid leukemia: predictors and associations with quality of life and functional status. Leukemia. 2007, 21:845-8. 10.1038/sj.leu.2404576

21. Wang XS, Giralt SA, Mendoza TR, et al.: Clinical factors associated with cancer-related fatigue in patients being treated for leukemia and non-Hodgkin's lymphoma. J Clin Oncol. 2002, 20:1319-28. 10.1200/JCO.2002.20.5.1319

22. Luskin M, Zhou E, Zhang Z, et al.: Sleep disturbance among patients with myelodysplastic syndromes and acute leukemia. Blood. 2019, 4786-4786. 10.1182/blood-2019-125147

23. Prieto JM, Blanch J, Atala J, Carreras E, Rovira M, Cirera E, Gastó C: Clinical factors associated with fatigue in haematologic cancer patients receiving stem-cell transplantation. Eur J Cancer. 2006, 42:1749-55. 10.1016/j.ejca.2005.10.005

24. Arcasoy MO: Anemia in the Patient with Cancer . Anemia. Benz Jr EJ, Berliner N, Schiffman FJ (ed): Cambridge University Press, UK; 2018 . 3:172. 10.1017/9781108586900.025

25. Banipal RP, Singh H, Singh B: Assessment of cancer-related fatigue among cancer patients receiving various therapies: a cross-sectional observational study. Indian J Palliat Care. 2017, 23:207. 10.4103/IJPC.IJPC_135_16

26. Mamolo C, Cappelleri J, Hoang C, et al.: A real-world, cross-sectional, community survey of symptoms and health-related quality of life of adults with acute myeloid leukemia. Future Oncol. 2019, 15:1895-909. 10.2217/fon-2018-0842

27. Albrecht TA, Boyiadzis M, Elswick Jr RK, Starkweather A, Rosenzweig M: Symptom management and psychosocial needs of adults with acute myeloid leukemia during induction treatment: a pilot study. Cancer nursing. 2017, 40:E31. 10.1097/NCC.0000000000000428

28. Tinsley SM, Sutton SK, Thapa R, Lancet J, McMillan SC: Treatment choices: a quality of life comparison in acute myeloid leukemia and high-risk myelodysplastic syndrome. Clin Lymphoma Myeloma Leuk. 2017, 17:S75-9. 10.1016/j.clml.2017.02.016

29. Kayastha N, Wolf SP, Locke SC, Samsa GP, El-Jawahri A, LeBlanc TW: The impact of remission status on patients' experiences with acute myeloid leukemia (AML): an exploratory analysis of longitudinal patientreported outcomes data. Support Care Cancer. 2018, 26:1437-45. 10.1007/s00520-017-3973-4

30. Alibhai SM, Leach M, Gupta V, Tomlinson GA, Brandwein JM, Saiz FS, Minden MD: Quality of life beyond 6 months after diagnosis in older adults with acute myeloid leukemia. Crit Rev Oncol Hematol. 2009, 69:16874. 10.1016/j.critrevonc.2008.07.015 